\title{
Recurso Hídrico Superficial en la Cuenca del río Tempisque, Costa Rica: Escenarios de Disponibilidad y Demanda
}

\section{Superficial Water Resource at Tempisque River Watershed, Costa Rica: Availability and Requirement Perspective}

Isabel Guzmán-Arias'

Fecha de recepción: 16 de agosto del 2013

Fecha de aprobación: 2 de octubre del 2013

Guzmán-Arias, I. Propuesta base para la planificación del recurso hídrico superficial en la cuenca del río Tempisque bajo escenarios de disponibilidad y demanda. Tecnología en Marcha. Número Especial Pág | | 8-| 32.

\footnotetext{
Doctora en Ciencias Naturales para el Desarrollo - Gestión de Recursos Naturales, máster en Educación Técnica, ingeniera agrícola, profesora e investigadora de la Escuela de Ingeniería Agrícola del Instituto Tecnológico de Costa Rica. Correo electrónico: iguzman@itcr.ac.cr
} 


\section{Isabel Guzmán Arias}

Isabel Guzmán Arias, nació el 18 de noviembre de 1973 en Cartago. Realizó sus estudios de primaria en la Escuela Monseñor Sanabria y de secundaria en el Colegio del Sagrado Corazón de Jesús, ambos en su ciudad natal.

En 1998 se graduó como Ingeniera Agrícola del Instituto Tecnológico de Costa Rica, luego se desempeñó como Ejecutiva de Ventas en una empresa dedicada a la venta de insumos agrícolas y sistemas de riego. Posteriormente trabajó como Encargada de Calidad Total en una compañía bananera.

En 2003 ingresó a laborar a la Escuela de Ingeniería Agrícola en el área de Manejo y Conservación de los Recursos Agua y Suelo, impartiendo cursos como Hidrología, Estadística Aplicada, Laboratorio de Hidráulica, entre otros.

En 2005 se graduó de la Maestría en Educación Técnica del Instituto Tecnológico de Costa Rica, lo que le permitió liderar el proceso de autoevaluación y acreditación de la Escuela de Ingeniería Agrícola ante la agencia de acreditación Canadian Engineering Acreditation Board.

Recientemente, en 2013 se graduó del Doctorado en Ciencias Naturales para el Desarrollo con Énfasis en Gestión de Recursos Naturales, título otorgado por la Universidad Nacional, Universidad Estatal a Distancia e Instituto Tecnológico de Costa Rica, donde pudo desarrollar como tema de tesis una "Base para la Planificación del Recurso Hídrico Superficial en la Cuenca Alta y Media del Río Tempisque".

Como investigadora ha tenido la oportunidad de participar y desarrollar proyectos como: "Evaluación de tres tipos de labranza en el cultivo de la papa (Solannum tuberosum)", finalizado en el 2009; "Valoración y Planificación del Recurso Hídrico en la Cuenca Alta y Canal Principal del Río Tempisque", finalizado en el 2012 y "Modelación Hidrodinámica y Morfológica del Río La Estrella", actualmente en ejecución.

El resultado de estas investigaciones ha sido ampliamente divulgado en diversos foros nacionales e internacionales, así como en la publicación de artículos científicos en revistas indexadas.

De esta manera ha podido participar en agrupaciones y actividades como:

Comité Técnico Asesor de Hidrometeorología y Ríos de la Comisión Nacional de Emergencias.

Comisión de Recurso Hídrico de la Municipalidad de Cartago.

Participación en los talleres para la elaboración de la Agenda del Agua en el 2012.

Participación en el taller para el Diseño de la Red Nacional de Monitoreo de Aguas Superficiales (Proyecto IWAVE)

Participación en el taller de consulta para la preparación del Decimonoveno Informe Estado de la Nación 2012 en el capítulo de Armonía con la Naturaleza en el tema de Recurso Hídrico.

En el ámbito familiar, está casada y tiene una hermosa hija de 12 años de edad. Sus actividades personales más importantes se centran en el crecimiento espiritual, la práctica de la natación y el disfrute de los acontecimientos familiares. 


\section{Palabras clave}

Disponibilidad; demanda; planificación; recurso hídrico; río Tempisque.

\section{Resumen}

Este artículo muestra la situación de disponibilidad y demanda del recurso hídrico en la cuenca alta y media del río Tempisque proyectada al 2030, y presenta las bases para un proceso de planificación del recurso. Los escenarios de disponibilidad de este incorporan las transformaciones en los flujos de agua por cambio en el uso de la tierra y por el cambio climático, cuyo efecto conjunto aumenta el problema de escasez en la época seca.

Los escenarios de demanda del recurso abarcan las proyecciones suministradas por los usuarios más importantes presentes en la cuenca, de los cuales se pueden vislumbrar muy pocas expectativas de crecimiento en términos de consumo de agua.

El proceso de planificación del recurso que se propone integra el análisis de esta tesis y trata de identificar las etapas que se deben seguir para un adecuado manejo del recurso en lo sucesivo.

\section{Key words}

Water availability; water demand; planning; water resources; Tempisque River.

\section{Abstract}

This paper describes the status of water resources availability and demand in the upper and middle Tempisque watershed projected up to 2030 and the proposed actions to start a planning process. The resource availability scenarios incorporate the modifications inwater flows due to land use and climate changes; these combined effects increases the problems of water shortages during the dry season.

The resource demand scenarios include projections provided by the major users in the watershed, of which very few can envision growth expectations in terms of water consumption.

The proposed resource planning process integrates the analysis conducted in this thesis and tries to identify the basic steps to be followed for the proper management of the resource in the future.

\section{Introducción}

El principal reto de esta investigación constituye el planteamiento de algunas líneas estratégicas viables de planificación del recurso hídrico, basadas en la recopilación de las experiencias de planificación, tanto en la cuenca del río Tempisque como en el resto del país y los demás países latinoamericanos. Este artículo define líneas estratégicas con una perspectiva provisoria que estima proyecciones futuras de las condiciones actuales, a saber: escenarios de cambio en el uso de la tierra, escenarios climáticos y escenarios de demanda del recurso. Sin duda, visualizar la vulnerabilidad, la amenaza y el riesgo del sistema hídrico de la cuenca del río Tempisque es el aporte más significativo de este estudio.

\section{Objetivo general:}

- Proponer una estrategia de planificación del recurso hídrico superficial para la cuenca alta y media del río Tempisque, basada en acciones específicas como medio de prevención de conflictos entre usuarios y atención a la sostenibilidad del recurso.

\section{Objetivos específicos:}

- Describir los escenarios de disponibilidad del recurso hídrico superficial en la cuenca para el 2010 y el 2030 bajo diferentes condiciones climáticas y de cambio en el uso de la tierra, según investigaciones realizadas.

- Generar escenarios de demanda del recurso hídrico superficial en la cuenca para el 2010 y el 2030.

- Construir un modelo base de acciones específicas de planificación del recurso hídrico congruente con la realidad fisiográfica, legal y política de la cuenca, así como con los escenarios de demanda del recurso hídrico. 


\section{Metodología}

Escenarios de disponibilidad del recurso hídrico

Para conocer el efecto del cambio climático y la transformación del uso de la tierra en la disponibilidad del recurso hídrico, se tomó como referencia el estudio de Calvo-Alvarado, J y Calvo, A. 20 I2. En él se seleccionó el modelo hidrológico para generar datos de caudal diario por medio de datos disponibles de lluvia, temperatura y humedad relativa diaria. El modelo empleado fue Hyluc, de la Universidad de Newcastle, uno de los pocos útiles para simular cambios de uso de la tierra en los flujos de la cuenca con base en información básica (Jewitt et al 2004, Calder et al 2003). Dado que el modelo utiliza datos de lluvia y temperatura, también sirvió para simular el impacto del cambio climático en los flujos de la cuenca.

\section{Escenarios de demanda del recurso hídrico}

La cuantificación de la demanda actual de recurso hídrico superficial se extrajo de la base de datos de concesiones de la Dirección de Aguas del MINAET, actualizados a mayo del 2012. Únicamente se utilizaron las concesiones de agua superficial y se clasificaron los caudales otorgados en cada concesión, de acuerdo con los diferentes usos.

En el caso de las proyecciones de demanda a futuro, se entrevistó a los representantes de los diferentes sectores usuarios más importantes y se les solicitó una estimación del caudal que requerirían para los siguientes 15-20, años con base en sus proyecciones de crecimiento. Los sectores entrevistados fueron:

- Sector agrícola

- Azucarera El Viejo.

- Central Azucarera del Tempisque CATSA.

- Arrocera El Pelón de la Bajura.

- Melones La Cueva.

- Servicio Nacional de Aguas Subterráneas, Riego y Avenamiento.

- Sector turismo

- Instituto Costarricense de Turismo.

- Sector agua potable

- Instituto Nacional de Acueductos y Alcantarillados.

- Concesiones

- Dirección de Aguas.

\section{Propuesta de planificación del recurso hídrico}

Se estableció una guía de trabajo para desarrollar una propuesta de acciones de planificación del recurso hídrico. Esta se elaboró por etapas; de ellas, la segunda se relaciona con el diseño de un modelo de planificación del recurso hídrico, y también se propone basado en el concepto de mayor participación de los sectores.

\section{Resultados y discusión}

\section{Escenarios de disponibilidad del recurso hídrico}

Según el estudio de Calvo-Alvarado y Calvo (20 I2), las condiciones de disponibilidad del recurso hídrico en la cuenca alta del río Tempisque se establecen al tomar como punto de menor altura el puente de Guardia. Para esta subcuenca se prevén variaciones significativas en los escenarios de disponibilidad del recurso hídrico para el 2030; uno de ellos está asociado al efecto del cambio en el uso de la tierra y el otro es el cambio climático.

En cuanto al cambio en el uso de la tierra, el impacto del aumento de la cobertura forestal en las áreas de conservación en el 2030 implica una disminución de los caudales en la estación lluviosa y un ligero aumento de los caudales de estiaje en la estación seca. En términos generales, el caudal medio anual disminuye en un 10,7 \% con respecto a los caudales medios registrados entre 1980 y 1985 . Esto se debe al aumento de las pérdidas de agua por evapotranspiración e intercepción de la cobertura forestal, que pasaría del $39 \%$ al 61 \% del área de la cuenca.

Un aspecto favorable del aumento de la cobertura es que influye positivamente al aumentar los flujos de estiaje durante la estación seca en porcentajes de entre un $9 \%$ y un $11 \%$ mensual con respecto a los flujos de estiaje del escenario 80-85 (CalvoAlvarado y Calvo, 20 I2).

A lo anterior se le ha adicionado el efecto de dos variables de cambio climático, como lo son el aumento de la temperatura en $2{ }^{\circ} \mathrm{C}$ y la disminución de la lluvia en un 10 \% para el 2030. Los estudios más recientes concluyen que tanto la variabilidad climática como la circulación general de la atmósfera experimentarán cambios significativos, que son los responsables de las variaciones 
en la precipitación anual y mensual observada en la simulación del clima futuro (ver Retana, 2012).

En la vertiente del Pacífico y en la zonanoroeste de Costa Rica, donde está ubicada la cuenca del río Tempisque, se estiman menos precipitaciones con respecto a las condiciones actuales. Se espera que las disminuciones sean mayores en el Pacífico Norte, luego en el Valle del General y déficits semejantes en el Valle Central y zona norte. La única excepción a este comportamiento deficitario se presentaría al sur de la Fila Costeña (Palmar Sur, Osa, Golfito, Coto, Burica), donde habría un incremento de precipitaciones. Por otra parte, las zonas más secas se relacionan con las zonas más cálidas durante el día (Retana, 2012).

La combinación de los escenarios anteriores da como resultado una reducción del caudal de escorrentía, porque aumenta la evaporación, la evapotranspiración y se reduce la entrada de agua por lluvia. El efecto es mayor en la estación lluviosa y ligeramente menor en la estación seca, toda vez que el aumento de la cobertura del bosque para el 2030 en las áreas de conservación amortigua el calentamiento en la época seca. En este escenario, la reducción del caudal medio anual es equivalente al 33 \% (Calvo-Alvarado y Calvo, 20 I2).

El cuadro I resume el impacto de ambos escenarios; en él se pueden observar tanto los porcentajes como los valores de caudales medios $(\mathrm{m} 3 / \mathrm{s})$ a nivel mensual y anual. Los dos escenarios pronostican una reducción de los caudales, excepto para los meses de la estación seca en el escenario de aumento del bosque.

En este caso, el caudal superficial, en los meses de estación lluviosa disminuye al aumentar la cobertura de bosque por el efecto de intercepción de este, pero a su vez eso favorece la recarga de agua subterránea; por eso en los meses de estación seca el caudal base aumenta al haber aporte de las reservas subterráneas. El efecto combinado de los dos escenarios es el que resulta crítico, dado que la reducción de los caudales es significativa, principalmente durante la estación seca.

Cuadro I. Impacto estimado del aumento de la cobertura forestal y el efecto del cambio climático (CC) en el caudal medio mensual y anual de la cuenca alta del río Tempisque inferido por el método Hyluc.

\begin{tabular}{|c|c|c|c|c|c|}
\hline \multirow[b]{2}{*}{ Mes } & \multicolumn{3}{|c|}{ Caudales medio $(\mathrm{m} 3 / \mathrm{s})$} & \multicolumn{2}{|c|}{ Diferencia con caudal 80-85 (\%) } \\
\hline & $\begin{array}{l}\text { Medido } \\
80-85\end{array}$ & $\begin{array}{c}\text { Ajustado } \\
\text { 2030+Bosque }\end{array}$ & $\begin{array}{c}\text { Ajustado } \\
2030+\text { Bosque }+C C\end{array}$ & 2030+Bosque & 2030+Bosque $+C C$ \\
\hline May & 29,94 & 22,84 & 17,08 & $-23,7$ & $-42,9$ \\
\hline Jun & 47,76 & 29,65 & 30,12 & $-17,0$ & $-36,9$ \\
\hline Jul & 28,47 & 24,65 & 16,90 & $-13,4$ & $-40,6$ \\
\hline Ago & 27,34 & 23,03 & $|5,8|$ & $-15,8$ & $-42,2$ \\
\hline Set & 56,44 & 49,62 & 37,69 & $-12,1$ & $-33,2$ \\
\hline Oct & 54,98 & 49,22 & 36,40 & $-10,5$ & $-33,8$ \\
\hline Nov & 37,56 & 37,11 & 28,34 & $-1,2$ & $-24,6$ \\
\hline Dic & 19,60 & 21,46 & 16,05 & 9,5 & $-18,1$ \\
\hline Ene & 15,22 & 16,94 & 12,72 & 11,3 & $-16,5$ \\
\hline Feb & 11,27 & 12,54 & 9,40 & 11,2 & $-16,6$ \\
\hline Mar & 8,54 & 9,51 & 7,13 & 11,4 & $-16,6$ \\
\hline Abr & 7,03 & 7,79 & 5,84 & 10,8 & $-16,9$ \\
\hline Año & 28,68 & 26,20 & 19,46 & $-10,7$ & $-33,7$ \\
\hline
\end{tabular}


Una vez expuestos estos escenarios de disponibilidad del recurso hídrico en la cuenca alta del río Tempisque, y si se considera el impacto de estos en los últimos meses de la época seca, necesariamente se debe cuantificar la demanda para visualizar el nivel de riesgo que esto significa y anteponerse a los eventuales conflictos que se pudieran suscitar. En el siguiente apartado se muestra el escenario de demanda.

\section{Escenarios de demanda del recurso hídrico}

Al igual que se construyeron escenarios para valorar el riesgo en la disponibilidad del recurso hídrico para el 2030, también se ha estimado una proyección de la demanda al tomar como base las expectativas de los usuarios más importantes en cuanto a crecimiento o disminución de cada sector.

Antes de estimar una proyección de la demanda, se hace un balance de las condiciones actuales para establecer un parámetro de comparación con las condiciones futuras.

\section{Demanda actual}

En la figura I se muestra la distribución de concesiones de agua superficial al 2012 en la cuenca alta del río Tempisque. En la figura 2 se muestra adicionalmente la distribución de concesiones en el tramo Guardia-La Guinea

Según la base de datos de concesiones de la Dirección de Aguas para mayo del 2012, en la cuenca alta se encontraban activas concesiones de agua superficial, por un caudal total de 7644,73 l/s, distribuidos en los usos que se muestran en el cuadro 2 .

Se aclara que las concesiones son otorgadas para un caudal determinado, pero no se lleva un registro de lo que realmente se utiliza mes a mes; por lo tanto, para efectos de los cálculos de balance "disponibilidad vs. demanda", para cada mes se considera el caudal total concesionado.

En el cuadro 3 se presenta el balance entre disponibilidad y demanda del recurso hídrico en la cuenca

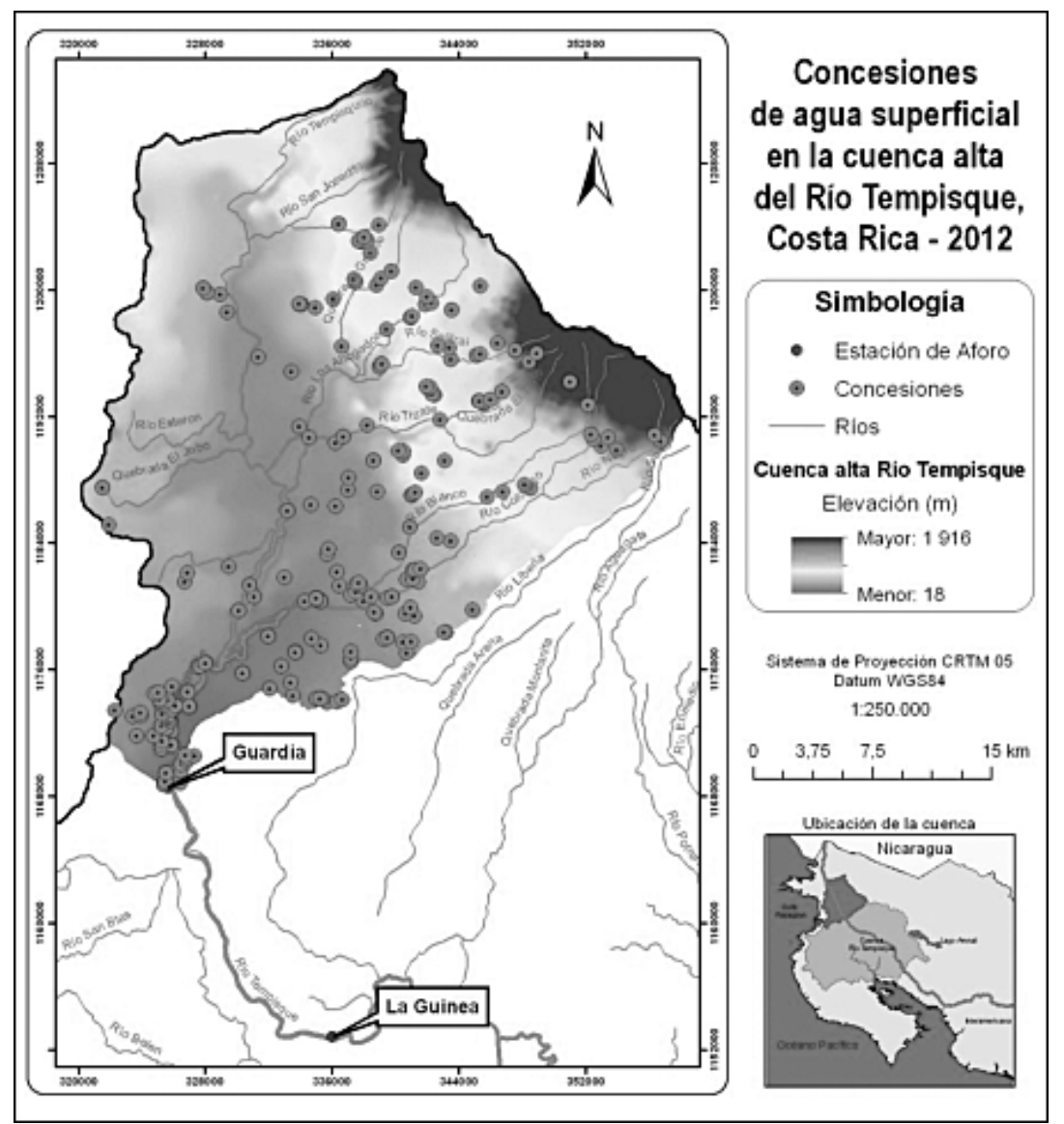

Figura I. Distribución de concesiones de agua superficial en la cuenca alta del río Tempisque, 2012. 


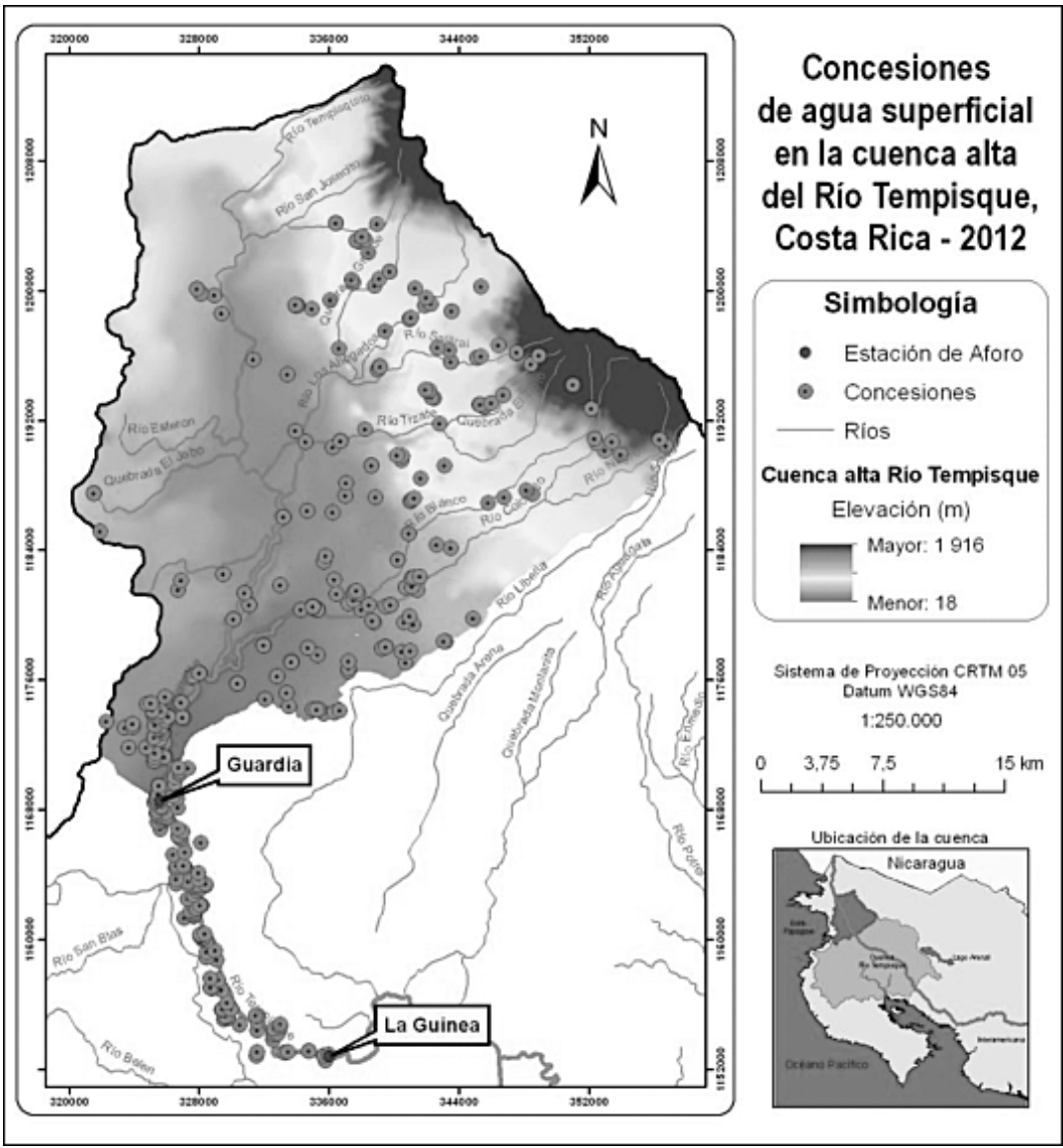

Figura 2. Distribución de concesiones de agua superficial en la cuenca alta del río Tempisque y el tramo Guardia- La Guinea para el 2012.

Cuadro 2. Caudales superficiales concesionados para los diferentes usos en la cuenca alta del río Tempisque (mayo 20।2)

\begin{tabular}{|c|c|}
\hline Tipo de uso & Caudal concesionado $(\mathrm{I} / \mathrm{s})$ \\
\hline Acueducto & 12,22 \\
\hline Agropecuario & 142,12 \\
\hline Consumo humano & 17,93 \\
\hline Industrial & 8,62 \\
\hline Riego & 7463,84 \\
\hline Total & 7644,73 \\
\hline
\end{tabular}

Fuente: Dirección de Aguas, 2012 alta del río Tempisque, cuya diferencia mensual permite conocer la disponibilidad real del recurso en el punto del puente de Guardia. Esta resultante constituye el caudal de oferta para satisfacer las necesidades entre los puntos de Guardia y La Guinea, y satisfacer el caudal ambiental. En el mismo cuadro se puede observar que el balance final es negativo. La presión de demanda que existe entre los puntos de Guardia y La Guinea se visualiza más fácilmente en la figura 2.

Específicamente, el uso del recurso hídrico clasificado por uso para toda la cuenca alta y el tramo Guardia-La Guinea se observa en el cuadro 4.

La realidad que se muestra al 2012 es ya demostrada como deficitaria. Según la Dirección de Aguas, la mayoría de estas concesiones ha llegado recientemente a su fecha de vencimiento y se han otorgado nuevamente por un periodo de diez años, lo cual significa que en ese periodo puede lograrse poco para proponer una reducción de los caudales otorgados. 
Cuadro 3. Balance de disponibilidad y demanda actual del recurso hídrico en la cuenca alta del río Tempisque al 20 I 2.

\begin{tabular}{|c|c|c|c|c|c|c|c|c|c|c|c|c|c|}
\hline \multirow{2}{*}{ Año 2012} & \multicolumn{13}{|c|}{ Caudal $\left(\mathrm{m}^{3} / \mathrm{s}\right)$} \\
\hline & Ene & Feb & Mar & $\mathrm{Abr}$ & May & Jun & Jul & Ago & Set & Oct & Nov & Dic & Anual \\
\hline Oferta & 15,22 & | I,27 & 8,54 & 7,03 & 29,94 & 47,76 & 28,47 & 27,34 & 56,44 & 54,98 & 37,56 & 19,60 & 28,68 \\
\hline Demanda & 7,64 & 7,64 & 7,64 & 7,64 & 7,64 & 7,64 & 7,64 & 7,64 & 7,64 & 7,64 & 7,64 & 7,64 & 7,64 \\
\hline $\begin{array}{l}\text { Oferta real } \\
\text { (puente } \\
\text { Guardia) }\end{array}$ & 7,58 & 3,63 & 0,9 & $-0,61$ & 22,3 & 40,12 & 20,83 & 19,7 & 48,8 & 47,34 & 29,92 & 11,96 & 21,04 \\
\hline $\begin{array}{c}\text { Demanda } \\
\text { Guardia-La } \\
\text { Guinea }\end{array}$ & 16,94 & 16,94 & 16,94 & 16,94 & 16,94 & 16,94 & 16,94 & 16,94 & 16,94 & 16,94 & 16,94 & 16,94 & 16,94 \\
\hline $\begin{array}{l}\text { Demanda } \\
\text { ambiental }\end{array}$ & 12,10 & 8,50 & 6,90 & 5,80 & 11,30 & 30,60 & 18,50 & 19,20 & 35,30 & 53,30 & 40,60 & 17,40 & \\
\hline Balance final & $-21,4$ & $-21,8$ & $-22,9$ & $-23,3$ & $-5,9$ & $-7,4$ & $-14,6$ & $-16,4$ & $-3,4$ & $-22,9$ & $-27,6$ & $-22,3$ & \\
\hline
\end{tabular}

Cuadro 4. Caudales superficiales concesionados para los diferentes usos en la cuenca alta y el tramo Guardia- La Guinea (mayo 20।2)

\begin{tabular}{|c|c|}
\hline Tipo de uso & $\begin{array}{c}\text { Caudal concesionado } \\
(\mathrm{I} / \mathrm{s})\end{array}$ \\
\hline Consumo humano & 1872,43 \\
\hline Agropecuario & 297,25 \\
\hline Riego agrícola & 21962,75 \\
\hline Turismo & 451,49 \\
\hline Total & 24583,92 \\
\hline
\end{tabular}

Fuente: Dirección de Aguas, 2012

\section{Demanda futura (2030)}

Las proyecciones de la demanda al 2030 se hacen para compararlas con los escenarios de disponibilidad del recurso hídrico bajo las condiciones antes descritas de cambio climático y transformación en el uso de la tierra.

Cabe aclarar que la demanda proyectada al 2030 se presenta numéricamente solo para la cuenca alta (ver cuadros 6 y 8); sin embargo, el análisis de crecimiento por sector usuario se hace al considerar también el tramo Guardia-La Guinea.
Como producto de entrevistas a los usuarios más representativos del recurso hídrico, a continuación se presentan las proyecciones de demanda al 2030, tanto en la cuenca alta como en el tramo GuardiaLa Guinea:

\section{- Uso agrícola}

En el uso agrícola, los actores principales son el cultivo del arroz, la caña de azúcar y, en menor medida, el melón. Para el caso del cultivo de arroz, el área de producción es de aproximadamente 5000 ha bajo el sistema de inundación abastecido desde diferentes fuentes de agua, entre las que se encuentra el río Tempisque, con una concesión de 700 l/s. Este cultivo en particular presenta características de precio en el mercado que no permiten proyectar crecimiento en los próximos años; por el contrario, los productores reconocen que podría darse una disminución del área de producción. No obstante, para efectos de este estudio se estimará la misma área de producción al 2030 para pronosticar el requerimiento hídrico (Soto, 2012)2.

En el caso del cultivo de caña de azúcar, existen dos productores principales en la cuenca del río Tempisque: Central Azucarera del Tempisque S.A.

2 Soto, A. 2012 Consumo de agua en el cultivo de arroz. El Pelón de la Bajura. Guanacaste. Comunicación personal. 
Cuadro 5. Sistemas de riego utilizados en el cultivo de la caña de azúcar.

\begin{tabular}{|c|c|c|c|c|}
\hline $\begin{array}{c}\text { Sistema de } \\
\text { riego }\end{array}$ & Eficiencia & I/s/ha & $\begin{array}{c}\text { Caudal } \\
\mathrm{m} 3 / \\
\text { ha*día }\end{array}$ & $\begin{array}{c}\mathrm{m} 3 / \\
\text { ha*mes }\end{array}$ \\
\hline Sifón & $39 \%$ & 2,23 & 192,31 & 5.77 \\
\hline Presurizado & $50 \%$ & 1,74 & 150,00 & 4.50 \\
\hline Pivote & $80 \%$ & 1,09 & 93,75 & 2.81 \\
\hline Goteo & $90 \%$ & 0,96 & 83,33 & 2.50 \\
\hline Aspersión & $45 \%$ & 1,93 & 166,67 & 5.00 \\
\hline
\end{tabular}

Fuente: Martínez, $2012^{3}$

(CATSA) y Azucarera El Viejo. Ambas empresas y sus respectivos productores independientes contabilizan más de 22000 ha en producción; aunque no toda esta área se abastece del río Tempisque, las concesiones otorgadas del río son de aproximadamente $6 \mathrm{~m}^{3} / \mathrm{s}$.

Los productores están conscientes de la importancia de evolucionar en sus sistemas de riego hasta lograr eficiencias de aplicación mucho más elevadas, y reconocen que esa sería la única opción de crecer en área de producción si se utilizan las mismas concesiones de agua con que cuentan. La inversión que se requiere para la transformación a sistemas de riego más eficientes es alta, por lo que es una alternativa que avanza lentamente. Los sistemas de riego utilizados en el cultivo de la caña de azúcar se exponen en el cuadro 5, donde se indica el porcentaje de eficiencia de aplicación para cada uno, así como el caudal requerido en cada caso.

Los sistemas de riego menos eficientes son el método de entrada al surco con sifón y el método por aspersión. El gasto en caudal es mucho más elevado con estos sistemas,precisamente porque la baja eficiencia en la aplicación exige agregar más agua para garantizar la lámina requerida por el cultivo.

Las proyecciones de crecimiento en área de producción de caña de azúcar están relacionadas con

3 Martínez, M. 2012 Consumo de agua en el cultivo de caña de azúcar. Azucarera El Viejo-Filadelfia, Guanacaste. Comunicación personal. el mejoramiento en la eficiencia de los sistemas de riego, de manera que puedan regar más área con la misma cantidad de agua concesionada (Campbell, 2012; Ponciano, 2012)4. En el caso de CATSA, ha comenzado a incrementar su área de producción en la parte alta de la cuenca del río Tempisque, específicamente en la finca conocida como Los Ahogados, con un aproximado de 600 ha, y podría seguir creciendo hacia este sector en los próximos años; no obstante, se estarían utilizando las concesiones existentes en la fincas según la actividad que se realice antes; probablemente sea ganadería.

En el caso del melón, el área de producción se ha reducido debido a las condiciones de competencia en el mercado y actualmente quedan tan solo 1500 ha con una concesión total sobre el río Tempisque de $200 \mathrm{l} / \mathrm{s}$ (Mendoza, 2012) ${ }^{5}$. Las proyecciones de crecimiento no se vislumbran a futuro, pero para efectos de este estudio se tomará como supuesto que permanezca igual en los próximos años.

\section{- Turismo}

Según las proyecciones del Instituto Costarricense de Turismo (ICT, 20I0), este sector cuenta con proyecciones de crecimiento al 2016 bajo las siguientes metas de crecimiento, calidad y sostenibilidad:

I. Aumento promedio del $5 \%$ en la cantidad de turistas internacionales con respecto al número de turistas que ingresaron en el 20 I0. Se calcula como una variación promedio del periodo 2010-2016, de lo cual se desprende que al 2016 se esperaría llegar a cerca de 2,7 millones de turistas internacionales.

2. Aumentar al menos a 500 empresas turísticas certificadas bajo la norma del programa de certificación para la sostenibilidad turística (CST). Se calcula partir de la línea base de 183 empresas certificadas en el 2010.

4 Campbell, B. 2012 Proyecciones de producción de caña de azúcar a futuro. Azucarera El Viejo. Guanacaste. Comunicación personal. Ponciano, A. 2012 Proyecciones de producción de caña de azúcar a futuro. Central Azucarera del Tempisque. Guanacaste. Comunicación personal.

5 Mendoza, A. 2012 Situación actual del cultivo de melón en Costa Rica. Compañía Del Monte. Guanacaste. Comunicación personal. promedio del periodo 2010-2016, de lo cual se desprende que al 2016 se esperaría llegar a cerca de 2,7 millones de turistas internacionales. 
3. Aumentar en un $10 \%$ el porcentaje de habitaciones hoteleras con declaratoria turística emitida por el ICT para que estas lleguen a representar cerca del $60 \%$ de la oferta nacional. Se calcula a partir de la línea base del $49 \%$ de las habitaciones declaradas al 2010.

En general, se espera que el crecimiento del sector al 2016 sea de un $3 \%$ anual y se esperaría que para el 2030, en el caso específico de Guanacaste, haya logrado estabilizarse, es decir, ya no habría más crecimiento (Lizano, 20I2) ${ }^{6}$. En la cuenca alta del río Tempisque no se registra ni se proyecta actividad turística.

\section{- Consumo humano}

El Instituto Costarricense de Acueductos y Alcantarillados (AyA) y los sistemas comunales están presentes en la distribución de agua para consumo humano en la cuenca del río Tempisque.

Los sistemas comunales (ASADAS, CAARS, municipalidades y otros) abastecen aproximadamente a 1,2 millones de habitantes en el país. Tres cuartas partes de los entes operadores atienden poblaciones menores a los 1000 habitantes, lo cual evidencia que en su mayoría se trata de sistemas sencillos desde el punto de vista operacional, pero mucho más complejos desde un punto de vista de gestión centralizada o aun regionalizada con los recursos existentes, lo cual obedece, principalmente, a su alta dispersión geográfica y a su naturaleza administrativa (Mora, 20 I I).

Por lo complejo que resulta obtener e integrar información sobre estos sistemas (por número y distribución), la subgerencia de sistemas comunales no tiene identificados cuáles han alcanzado su capacidad de producción máxima, ya que aún no existe una base de datos especializada que permita visualizar la ubicación y el estado de todos los entes operadores; esto dificulta proyectar la capacidad de estos sistemas a futuro para abastecer una demanda creciente para consumo humano.

Todos los requerimientos de inversión identificados mediante la iniciativa de la Subgerencia de Ambiente, Investigación y Desarrollo (SAID) no podrían materializarse, aun si hubiera financiamiento,

6 Lizano, R. 2012 Proyecciones de crecimiento para el sector turístico en Guanacaste. ICT. Comunicación personal. si primero no se garantizan el acceso y la conservación del recurso hídrico. La SAID tiene clara la necesidad de estudiar a profundidad y con detalle este recurso para poder avanzar en el proceso de planificación del recurso hídrico, tema aún pendiente en Costa Rica (Mora, 20I I).

En todo caso, si se considera el requerimiento de caudal para consumo humano en el 2012 en la cuenca alta del río Tempisque, el cual es de 17,9 $1 / s$, y si se sabe que el consumo por persona es en promedio de 350 I diarios, se puede conocer la población correspondiente a este consumo como 4426 personas. Al hacer una proyección de consumo al 2030 y tomar como base las proyecciones de población de INEC 2008, se estima que la población antes mencionada crecería a 5395 personas, para un consumo de 21,9 l/s. Este valor corresponde a caudal superficial, toda vez que el uso del agua para consumo humano es extraído principalmente de las reservas subterráneas mediante pozos.

En síntesis, la proyección en la demanda del recurso hídrico en la cuenca alta para el 2030, considerando los diferentes usos, se muestra numéricamente en el cuadro 6.

- Uso ilegal

Tanto al escenario de demanda del 2012 como del 2030 se les debe agregar el uso ilegal del recurso hídrico tanto por extracciones que se realicen sin concesión como por dueños de concesiones que eventualmente podrían estar extrayendo más caudal del concedido. Estas extracciones ilegales de agua se han estimado, según la Dirección de Aguas ${ }^{7}$, como un $20 \%$ de las concesiones otorgadas en forma legal (ver cuadro 7).

El balance de disponibilidad y demanda para el 2030 incluyendo los escenarios de cambio en el uso de la tierra y cambio climático se muestra en el cuadro 8 para la cuenca alta hasta el Puente de Guardia.

La información del cuadro 8 revela un balance final en números negativos ya desde el punto del puente de Guardia, lo cual significa que si se agregara a partir de ahí la demanda que existe hasta el punto de la Guinea, tal y como se hizo con el escenario del 2012, no habría ningún caudal por distribuir.

7 Zeledón, J.M. 2012. Demanda actual y futura de agua en el río Tempisque. Dirección de Aguas. MINAET. Comunicación personal. 
Cuadro 6. Caudales superficiales concesionados para los diferentes usos en la cuenca alta del río Tempisque (proyección 2030)

\begin{tabular}{|c|c|}
\hline Tipo de uso & $\begin{array}{c}\text { Caudal concesionado } \\
(1 / \mathrm{s})\end{array}$ \\
\hline Acueducto & 12,22 \\
\hline Consumo humano & 21,86 \\
\hline Industrial & 8,62 \\
\hline Riego & 7605,96 \\
\hline Total & 7648,66 \\
\hline
\end{tabular}

Bajo estas condiciones no queda más alternativa que trabajar en un modelo de planificación que pueda ser ejecutado desde el mismo momento en que se cumplan los diez años de validez de las concesiones recientemente renovadas.

\section{Etapas para la planificación del recurso hídrico}

En la cuenca del río Tempisque, al igual que en el resto del país, aún se evidencia la falta de objetivos claros sobre cómo manejar los recursos de manera sostenible. El tema de planificación del recurso hídri- co todavía está ausente en las grandes decisiones políticas de Costa Rica; pese a los grandes esfuerzos por contar con los mecanismos de manejo del recurso hídrico, muchos de ellos están muy bien planteados en documentos, pero por múltiples razones no se logran llevar con éxito a la ejecución.

Actualmente, al igual que lo manifestó Echeverría (2005), la gestión de los recursos hídricos en la cuenca del río Tempisque continúa siendo de forma sectorial, de acuerdo con la visión y la necesidad de cada actor. No se cuenta con un plan bien estructurado que haya surgido de un proceso de diagnóstico participativo y debidamente dirigido por un ente responsable.

Por lo tanto, se plantea una propuesta de planificación del recurso hídrico para la cuenca del río Tempisque, que abarque las fases del proceso que van desde la concepción misma del modelo hasta su normalización para ser reproducido en otras cuencas, tal y como se muestra en el diagrama de proceso de la figura 3.

El enfoque de esta propuesta se basa en el modelo implementado en México, el cual se caracteriza por desarrollarse de abajo hacia arriba, partiendo de la percepción local e integrándola en la concepción

Cuadro 7. Extracciones ilegales de caudal superficial en la cuenca alta para el 2012 y el 2030.

\begin{tabular}{|c|c|c|c|}
\hline $\begin{array}{c}\text { Caudal concesionado } \\
2012(1 / 5)\end{array}$ & $\begin{array}{c}\text { Caudal concesionado } \\
\text { proyectado al 2030 (1/s) }\end{array}$ & $\begin{array}{c}\text { Caudal ilegal 2012 } \\
(1 / 5)\end{array}$ & $\begin{array}{c}\text { Caudal ilegal } \\
\text { proyectado al 2030 (I/s) }\end{array}$ \\
\hline 7644,73 & 7648,66 & 1528,95 & 1529,73 \\
\hline
\end{tabular}

Cuadro 8. Balance de disponibilidad y demanda actual del recurso hídrico en la cuenca alta de río Tempisque para el 2030.

\begin{tabular}{|c|c|c|c|c|c|c|c|c|c|c|c|c|c|}
\hline \multirow{2}{*}{ Año 2030} & \multicolumn{13}{|c|}{ Caudal $\left(\mathrm{m}^{3} / \mathrm{s}\right)$} \\
\hline & Ene & Feb & Mar & $\mathrm{Abr}$ & May & Jun & Jul & Ago & Set & Oct & Nov & Dic & Anual \\
\hline Oferta & 12,72 & 9,4 & 7,13 & 5,84 & 17,08 & 30,12 & 16,9 & $|5,8|$ & 37,69 & 36,4 & 28,34 & 16,05 & 19,46 \\
\hline Demanda & 7,64 & 7,64 & 7,64 & 7,64 & 7,64 & 7,64 & 7,64 & 7,64 & 7,64 & 7,64 & 7,64 & 7,64 & 7,64 \\
\hline $\begin{array}{c}\text { Oferta } \\
\text { real (puente } \\
\text { Guardia) }\end{array}$ & 5,08 & 1,76 & $-0,5$ I & $-1,8$ & 9,44 & 22,48 & 9,26 & 8,17 & 30,05 & 28,76 & 20,7 & 8,41 & 11,82 \\
\hline $\begin{array}{l}\text { Demanda } \\
\text { ambiental }\end{array}$ & 12,1 & 8,5 & 6,9 & 5,8 & 11,3 & 30,6 & 18,5 & 19,2 & 35,3 & 53,3 & 40,6 & 17,4 & \\
\hline Balance final & $-7,02$ & $-6,74$ & $-7,41$ & $-7,6$ & $-1,86$ & $-8,12$ & $-9,24$ & $-11,03$ & $-5,25$ & $-24,54$ & $-19,9$ & $-8,99$ & \\
\hline
\end{tabular}




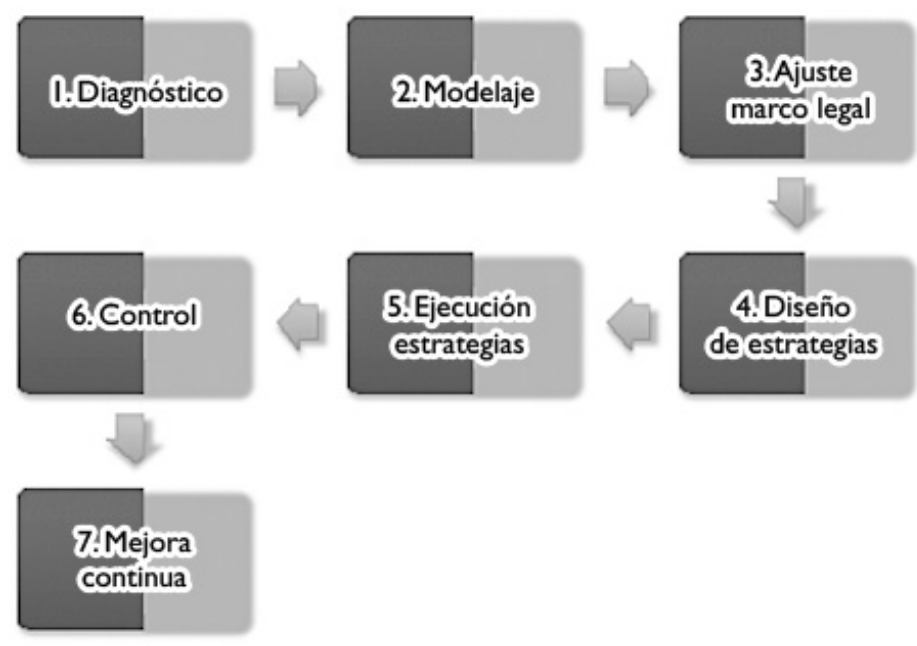

Figura 3. Guía de trabajo para la planificación del recurso hídrico en la cuenca alta y media del río Tempisque

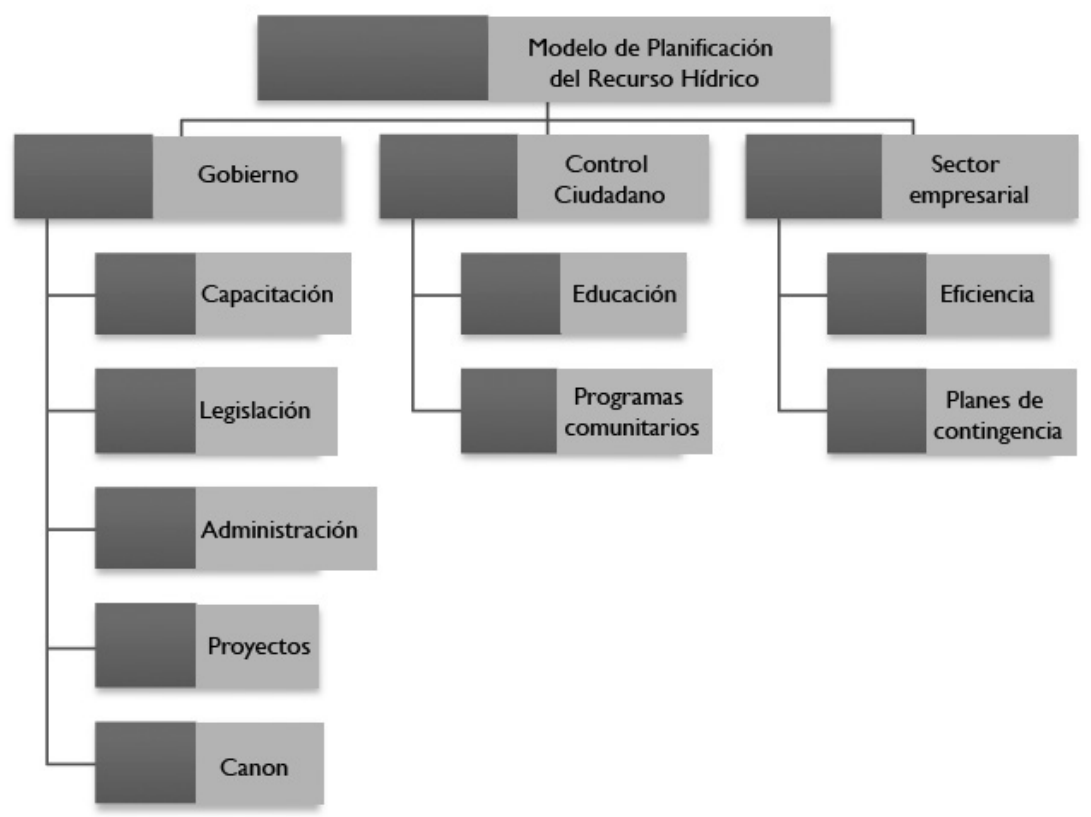

Figura 4. Modelo de planificación del recurso hídrico.

del modelo como parte de los estudios de diagnóstico, para así garantizar su incorporación en el plan de manejo del recurso hídrico.

\section{Objetivos de cada fase}

Fase diagnóstico: elaborar el diagnóstico con posibles escenarios y necesidades presentadas por los actores, así como la recopilación de experiencias de planificación del recurso hídrico en la cuenca del río Tempisque, en el país y el resto de países de América Latina.

Fase modelaje: diseñar un modelo de planificación del recurso hídrico para la cuenca alta y media del río Tempisque, que sea sencillo de ejecutary fácil de adaptar a otras cuencas.

Fase ajuste legal: armonizar la propuesta de planificación con el marco legal e institucional que cubre el tema de recurso hídrico. 
Fase diseño de estrategias: diseñar las estrategias por seguir, derivadas del modelo de planificación y que permitan la gestión integral del recurso hídrico.

Fase ejecución de estrategias: llevar a vabo las estrategias mediante acciones concretas, con la participación de los actores involucrados.

Fase control: ajustar los elementos del modelo de planificación que se hayan detectado con algún tipo de problema durante la fase de ejecución o monitoreo.

Fase mejoramiento: ejecutar el modelo ajustado en la fase anterior. Modelo base de planificación del recurso hídrico: el modelo de planificación se enfoca en las dimensiones de participación de los diferentes sectores, según las potencialidades de cada uno, tal y como se muestra en la figura 4.

El modelo propone la participación equitativa de tres dimensiones: gobierno, ciudadanía y sector empresarial, y la articulación de los elementos que son comunes para el logro de los productos desea- dos. Para comprender mejor el modelo se desglosan los objetivos y productos correspondientes a cada uno de esos componentes.

- Componente gobierno (Cuadro 9)

- Componente control ciudadano (Cuadro 10)

- Componente sector empresarial (Cuadro II)

Al haber participación de tres sectores claramente definidos, se esperaría que la responsabilidad de dirigir el proceso y posteriormente ejecutar las acciones sea un consejo de cuenca con las facultades necesarias para organizar la participación de la sociedad en la gestación y ejecución del plan.

Los consejos de cuenca tienen un buen referente de éxito en los esfuerzos que está llevando a cabo México en el tema de la planificación y gestión del recurso hídrico.

Los escenarios de disponibilidad del recurso hídrico revelan una eventual escasez de agua de nivel crítico para el 2030 en la época seca.

Cuadro 9. Componente gobierno.

\begin{tabular}{|c|c|c|}
\hline Variable & Objetivo & Productos esperados \\
\hline Capacitación & $\begin{array}{l}\text { - Comprender el sistema hídrico de la cuenca para } \\
\text { mejorar los criterios de toma de decisiones. }\end{array}$ & $\begin{array}{l}\text { - Un programa de capacitación en diferentes temas } \\
\text { relacionados con los sistemas hídricos, con la } \\
\text { participación de representantes de las instituciones } \\
\text { relacionadas con el recurso hídrico. }\end{array}$ \\
\hline Legislación & $\begin{array}{l}\text { - Contar con los mecanismos legales para una } \\
\text { gestión eficiente y efectiva del recurso hídrico. }\end{array}$ & $\begin{array}{l}\text { - Leyes adecuadas a los requerimientos actuales del } \\
\text { recurso hídrico y su sostenibilidad. }\end{array}$ \\
\hline \multirow{3}{*}{ Administración } & $\begin{array}{l}\text { - Articular adecuadamente los campos de acción } \\
\text { de cada una de las instituciones de gobierno } \\
\text { relacionadas con el agua. }\end{array}$ & $\begin{array}{l}\text { - Estudio de diagnóstico de los campos de acción } \\
\text { de cada uno de las instituciones para detectar } \\
\text { traslapes y vacíos en las funciones. }\end{array}$ \\
\hline & $\begin{array}{l}\text { - Dotar a la dirección de aguas de los mecanismos } \\
\text { y recursos para mejorar la administración del } \\
\text { recurso hídrico mediante las concesiones. }\end{array}$ & $\begin{array}{l}\text { - Reajuste concesiones de agua y control de los } \\
\text { consumos por parte de los usuarios. }\end{array}$ \\
\hline & $\begin{array}{l}\text { Establecer un organismo de cuenca que vele } \\
\text { por el desarrollo del modelo de planificación del } \\
\text { recurso hídrico. }\end{array}$ & - Organismo de cuenca en funcionamiento. \\
\hline Proyectos & $\begin{array}{l}\text { - Visualizar oportunidades de proyectos que puedan } \\
\text { contribuir con el aprovechamiento del recurso. }\end{array}$ & $\begin{array}{l}\text { - Propuestas de almacenamiento de agua en época } \\
\text { Iluviosa para su utilización en época seca. } \\
\text { - Puesta en operación del proyecto Piedras. }\end{array}$ \\
\hline Canon & $\begin{array}{l}\text { - Demostrar la inversión producto del canon. } \\
\text { - Revisar el canon con el fin de ajustar los montos a } \\
\text { cobrar y mejorar los incentivos. }\end{array}$ & $\begin{array}{l}\text { - Obras de inversión para el mejoramiento en el } \\
\text { servicio de distribución. } \\
\text { - Canon mejorado. }\end{array}$ \\
\hline
\end{tabular}


Cuadro 10. Componente control cuidadano.

\begin{tabular}{|c|c|c|}
\hline Variable & Objetivo & Productos esperados \\
\hline Educación & $\begin{array}{l}\text { - Introducir el tema de sostenibilidad del recurso } \\
\text { hídrico en los niveles de preescolar, primaria y } \\
\text { secundaria. } \\
\text { Desarrollar campañas educativas para la } \\
\text { población en general. }\end{array}$ & $\begin{array}{l}\text { - Tema del recurso hídrico en los planes de } \\
\text { estudio. } \\
\text { - Dos campañas anuales para educar a la } \\
\text { población. }\end{array}$ \\
\hline $\begin{array}{l}\text { Programas } \\
\text { Comunitarios }\end{array}$ & $\begin{array}{l}\text { Desarrollar programas comunitarios de } \\
\text { protección del recurso hídrico. }\end{array}$ & $\begin{array}{l}\text { - Una comunidad modelo con el programa } \\
\text { completamente implementado. } \\
\text { - Involucramiento de otras comunidades dada la } \\
\text { experiencia de la comunidad modelo. }\end{array}$ \\
\hline
\end{tabular}

Cuadro I I. Componente sector empresarial

\begin{tabular}{|l|l|l|}
\hline \multicolumn{1}{|c|}{ Variable } & \multicolumn{1}{|c|}{ Objetivo } & \multicolumn{1}{|c|}{ Productos esperados } \\
\hline Eficiencia & $\begin{array}{l}\text { Mejorar los sistemas de conducción y distribución } \\
\text { del agua. }\end{array}$ & $\begin{array}{l}\text { Sistemas de conducción y sistemas de riego que } \\
\text { garanticen alta eficiencia. }\end{array}$ \\
\hline $\begin{array}{l}\text { Planes de } \\
\text { contingencia }\end{array}$ & $\begin{array}{l}\text { Solicitar un plan de contingencia en caso de escasez } \\
\text { como requisito para otorgar una concesión de agua. }\end{array}$ & $\begin{array}{l}\text { Documento con plan de contingencia de cada } \\
\text { empresa. }\end{array}$ \\
\hline
\end{tabular}

Los escenarios de demanda muestran cierta estabilidad en la cantidad de agua requerida, toda vez que no se proyectan grandes crecimientos en las diferentes actividades productivas y de consumo.

A pesar de que las concesiones no se encuentran en el mismo estado de aprovechamiento al mismo tiempo, debido a los periodos de vencimiento y renovación, sí existe un máximo de demanda en los meses de época seca, por coincidir con la época de mayor requerimiento de agua en los cultivos de arroz, caña de azúcar y melón.

La propuesta de planificación del recuso hídrico adapta el enfoque del modelo mexicano con una participación de abajo hacia arriba, es decir, involucrando la percepción de la sociedad local y simultáneamente activando la participación del gobierno, el control ciudadano y sector empresarial, mediante un consejo de cuenca.

\section{Recomendaciones}

El modelo de planificación requiere de un proceso de validación antes de llevarlo a la ejecución.
Es importante insistir en la conformación de un consejo de cuenca y establecer una relación de coordinación de acciones entre los usuarios y la Dirección de Aguas.

Este estudio puede ser utilizado como insumo para las próximas decisiones por tomar en el tema del recurso hídrico.

\section{Bibliografía}

Calder, I. R., I. Reid, T. R. Nisbet, and J. C. Green. 2003. Impact of lowland forests in England on water resources: Application of the Hydrological Land Use Change (HYLUC) model, Water Resource. Res., 39( I I).

Calvo-Alvarado, J. y Calvo, A. 2012 Estimación del impacto del cambio del uso de la tierra y el clima en los caudales medios de la cuenca alta del Río Tempisque, Costa Rica.9p.

Dirección de Aguas - MINAET (Ministerio de Ambiente, Energía y Telecomunicaciones). 2012. Base de Datos de Concesiones.

Echeverría, J. 2005 Estudio de caso cuenca Tempisque, Costa Rica. Estrategia de Gestión Integrada del Recurso Hídrico. MINAE. Costa Rica, 20 p.

ICT (Instituto Costarricense de Turismo) 20I0. Plan Nacional de Turismo Sostenible de Costa Rica 2010 - 2016. 188 p. 
INEC (Instituto Nacional de Estadística y Censos), 2008. Estimaciones y Proyecciones de Población por Sexo y Edad (cifras actualizadas) 1950 - 2050. San José, 143 p.

Jewitt G; Garratt, J; Calder, I and L.Fuller. 2004. Water resources planning and modeling tools for the assessment of land use change in the Luvurhu Catchment, South Africa Physics and Chemistry of the Earth (29) |233-1241.

MINAE (Ministerio de Ambiente y Energía), 2006. Canon de aprovechamiento de aguas. Costa Rica. 13 p.
Mora, G. 20I I Costa Rica: necesidades de inversión del sector agua potable y alcantarillado sanitario. Hidrogénesis 9(I): 47- 63

SENARA (Servicio Nacional de Aguas Subterráneas, Riego y Avenamiento) 2010

Estudio de Factibilidad, Diseño Preliminar y Documentos de Licitación para la Construcción de la Presa Embalse DRAT. $84 \mathrm{p}$.

Retana, J. 2012. Riesgo Futuro del Sector Hídrico de Costa Rica ante el Cambio Climático. IMN (Instituto Meteorológico Nacional) 20 p. 